\title{
Sex Pheromone of the Black Cutworm Moth, Agrotis ipsilon HufNaGel (Lepidoptera: Noctuidae): Attractant Synergist and Improved Formulation ${ }^{1}$
}

\author{
Sadao Wakamura, Dean L. Struble, ${ }^{2}$ Hiroichi Matsuura, ${ }^{3,4}$ \\ Masataro SATO ${ }^{5,6}$ and Kazuo KegASAwA ${ }^{7}$ \\ Shikoku National Agricultural Experiment Station, Zentsuji, Kagawa 765, Japan \\ ${ }^{2}$ Agriculture Canada Research Station, Lethbridge, Alberta, Canada T1J 4B1 \\ ${ }^{4}$ Ishikawa Prefectural Agricultural Experiment Station, Nonoichi, Ishikawa 921, Japan \\ ${ }^{6}$ Shonai Branch, Yamagata Prefectural Agricultural Experiment Station, \\ Fujishima, Yamagata 999-76, Japan
}

(Received September 20, 1985)

\begin{abstract}
Attractive activity of a 3:1 mixture of ( $Z$ )-7-dodecenyl acetate (Z7-12:Ac) and $(Z)$ 9-tetradecenyl acetate (Z9-14:Ac) for male black cutworm moths, Aqrotis ipsilon Hufnagel, was significantly improved by the addition of $(Z)$-11-hexadecenyl acetate (Z11-16: Ac) when dispensed from a rubber septum. On the contrary, the addition of Z11-16: Ac caused a significant decrease of catches when dispensed from a glass capillary tube. The reason was not known. Nevertheless, a rubber septum impregnated with $30 \mu \mathrm{g}$ of Z7-12:Ac, $10 \mu \mathrm{g}$ of Z9-14: Ac and 150 $\mu \mathrm{g}$ of Z11-16: Ac was one of the most effective lures for male black cutworm moths. This formulation was effective for 1 month or more after a 10-day pre-exposure.
\end{abstract}

\section{INTRODUCTION}

The black cutworm, Agrotis ipsilon Hufnagel, is a serious pest on many vegetables, soybean, forage crops, and so forth. The black cutworm sometimes has repeated sudden outbreaks especially on forage crops in Japan (OHMORI and Hasegawa, 1968; etc.), so that an early warning of incidence is needed for preparation of appropriate control against this pest.

The sex pheromone of $A$. ipsilon was identified as $(Z)$-7-dodecenyl acetate (Z7-12:Ac) and $(Z)$-9-tetradecenyl acetate (Z9-14:Ac), and two formulations were presented as the most effective lures by HiLl et al. (1979); $30 \mu \mathrm{g}$ of Z7-12:Ac plus $10 \mu \mathrm{g}$ of Z9-14: Ac on a rubber septum, and a 3:1 mixture of Z7-12:Ac and Z9-14:Ac dispensed from a $0.2-\mathrm{mm}$-ID glass capillary tube sealed at one end. In our preliminary investigations conducted in Japan, however, fewer males were caught with these formulations than with a blacklight trap (unpublished data). The addition of $(Z)$-11-hexadecenyl acetate

1 A part of this work was presented at the Annual Meeting of Japanese Society of Applied Entomology and Zoology (April, 1982, Nagoya).

3 Present address: National Agriculture Research Center, Yatabe, Ibaraki 305, Japan

5 Present address: Shonai Plant Protection Office, Mikawa, Yamagata 997-13, Japan

7 Present address: Tohoku National Agricultural Experiment Station, Morioka 020-01, Japan 
(Z11-16:Ac) to the 2-component blend was found to increase the catches of A. ipsilon males in preliminary tests done in Canada, Switzerland and New Zealand (unpublished data, D. L. Struble, G. L. Ayre, E. Städler and R. A. Galbreath).

This paper describes the effects of the third compound on the attraction of male A. ipsilon in Japan.

\section{MATERIALS AND METHODS}

Chemicals. Z7-12:Ac and Z9-14:Ac were obtained from the Farchan Chemical Company and the Takeda Chemical Industry, Ltd., respectively. Z11-16:Ac was provided by Dr. S. Takahashi, Pesticide Research Institute, Kyoto University. Each compound was purified with column chromatography on silicic acid impregnated with $16.7 \% \mathrm{AgNO}_{3}$. The compounds were more than $99 \%$ pure with respect to positional and geometrical isomers. Hexadecyl acetete (16:Ac) and tetradecyl acetate (14:Ac) were synthesized from the corresponding alcohols.

The chemicals were evaporated from rubber septa or glass capillary tubes sealed at one end. Chemicals were dissolved in distilled hexane and the appropriate volume was placed in the rubber septa (Daburu Kyappu, No. 2, Araki Rubber Co. Ltd.), and then the solvent was allowed to evaporate. The septa were stored in vials at $-20^{\circ} \mathrm{C}$ till use. The glass capillary tubes used were 5-, 10-, 20- and 50- $\mu l$ Micropet ${ }^{\circledR}$ disposable micropippettes (0.266, 0.376, 0.532, and 0.841 mm ID, respectively; Becton, Dickinson and Company). Chemicals were mixed and the tubes were filled to a depth of about $1 \mathrm{~cm}$ by the following procedure described by Kozai and Wakamura (1982). The tube, containing a $1-\mathrm{cm}$ column of liquid at the middle point, was sealed at one end by heating. Then the liquid column was moved to the sealed end by centrifugation at 1,500 rpm for $5 \mathrm{~min}$. The open end was then sealed by heating and the tube was stored at room temperature till use. Immediately before use the tube was cut to leave a $1-\mathrm{cm}$ space between the liquid and the open surface.

Field tests. Several water-pan traps $(30 \times 24 \times 15(\mathrm{~h}) \mathrm{cm}$, Takeda Chemical Industry, Ltd.) were set at $1-\mathrm{m}$ above the ground and more than $20-\mathrm{m}$ intervals. Tests were conducted at Zentsuji, Kagawa from 1981 to 1984, at Nonoichi, Ishikawa in 1982 and 1983, and at Fujishima, Yamagata in 1984. In certain tests conducted at Zentsuji, males obtained from the laboratory culture were released with markings of oily dye on their forewings.

Trap locations were rearranged at appropriate intervals in order to avoid any effect dependent on trap locations. Catches of male moths $(X)$ were transformed to $\sqrt{X+0.5}$, submitted to an analysis of variance and ranked by Duncan's new multiple range test (DUNCAN, 1955). In the tables, values followed by the same letter are not significantly different at $5 \%$ level.

\section{RESULTS AND DISCUSSION}

The 3:1 mixture of Z7-12:Ac and Z9-14:Ac at $40 \mu \mathrm{g} /$ rubber septum containing $150 \mu \mathrm{g}$ of Z11-16:Ac, 16:Ac, or 14:Ac was tested to determine whether the relatively large amount of a third compound would improve the catches of moths by altering the release rate of Z7-12:Ac and Z9-14:Ac from the rubber septum (Table 1). The catches of moths with rubber septum containing Z11-16:Ac were greater than with the two- 
Table 1. Catches of male black cutworm moths with rubber septa impregnated with a 3:1 mixture of Z7-12:Ac and Z9-14: Ac containing Z11-16:Ac, 16:Ac or 14:Ac (Zentsuji, May 5-26, 1981)

\begin{tabular}{cccc}
\hline \multicolumn{2}{c}{$\mu$ g of compound added/septum ${ }^{\mathrm{a}}$} & \multicolumn{2}{c}{ Total no. of males caught $^{\mathrm{b}}$} \\
\cline { 1 - 3 } Z11-16: Ac & $16:$ Ac & $14:$ Ac & $1 \mathrm{~b}$ \\
\hline 0 & 0 & 0 & $24 \mathrm{a}$ \\
0 & 0 & 0 & $4 \mathrm{~b}$ \\
0 & 150 & 0 & $3 \mathrm{~b}$ \\
Glass capillary tube & 0 & 150 & $8 \mathrm{~b}$ \\
\hline
\end{tabular}

a Basic formulation:30 $\mu \mathrm{g}$ of Z7-12:Ac+10 $\mu \mathrm{g}$ of Z9-14:Ac/septum.

b Values indicated are the total for each 1 trap throughout the test period. Values with the same letter are not significantly different by Duncan's new multiple range test at $5 \%$ level.

c A 0.266-mm ID tube dispensing a 3:1 mixture of Z7-12:Ac and Z9-14:Ac.

Table 2. Catches of male black cutworm moths with rubber septa impregnated with Z7-12:Ac, Z9-14:Ac and Z11-16:Ac (Nonoichi, April 25-July 5 and Zentsuji, April 20-May 23, 1983)

\begin{tabular}{|c|c|c|c|c|}
\hline \multicolumn{3}{|c|}{$\mu \mathrm{g}$ of compound/septum } & \multicolumn{2}{|c|}{ Total no. of males caught } \\
\hline $\mathrm{Z7}-12: \mathrm{Ac}$ & Z9-14:Ac & $\mathrm{Z} 11-16: \mathrm{Ac}$ & Nonoichi & Zentsuji \\
\hline 30 & 10 & 0 & $10 \mathrm{~b}$ & $1 \mathrm{~b}$ \\
\hline 30 & 10 & 15 & $289 \mathrm{a}$ & $10 \mathrm{a}$ \\
\hline 30 & 10 & 50 & $266 \mathrm{a}$ & $14 \mathrm{a}$ \\
\hline 30 & 10 & 150 & $419 \mathrm{a}$ & $13 \mathrm{a}$ \\
\hline 30 & 10 & 500 & - & $1 \mathrm{~b}$ \\
\hline \multicolumn{3}{|c|}{ Glass capillary tube ${ }^{b}$} & - & $1 \mathrm{~b}$ \\
\hline \multicolumn{3}{|l|}{ Light trape } & $13 \mathrm{~b}$ & - \\
\hline \multicolumn{5}{|c|}{$\begin{array}{l}\text { a The values indicated are the total for each } 1 \text { trap throughout test period, and the values } \\
\text { followed by the same letter are not significantly different by DuncAN's new multiple range test } \\
\text { at } 5 \% \text { level. }\end{array}$} \\
\hline \multicolumn{5}{|c|}{$\begin{array}{l}\text { A } 0.266-\mathrm{mm}-\mathrm{ID} \text { tube dispensing a } 3: 1 \mathrm{mixtu} \\
\text { A mercury-lamp trap for forecasting. }\end{array}$} \\
\hline
\end{tabular}

component blend of Z7-12:Ac and Z9-14:Ac on a rubber septum or in a glass capillary tube. Neither the addition of 16:Ac nor 14:Ac caused a significant increase in catches. These resuts showed that Z11-16:Ac acted as a synergist for the attraction of males and that the improvement of catches by altering the release rate of the components did not occur. The following tests were conducted to evaluate the synergistic activity of Z11-16:Ac.

The 3:1 mixture of Z7-12:Ac and Z9-14:Ac at $40 \mu \mathrm{g} / \mathrm{septum}$ plus 15 to $500 \mu \mathrm{g}$ of Z11-16: Ac was tested to determine the optimal dose of Z11-16:Ac (Table 2). The catches of males with the septa containing 15 to $150 \mu \mathrm{g}$ of Z11-16: Ac were significantly greater than with the septum containing no Z11-16:Ac. The addition of $500 \mu \mathrm{g}$ of Z11-16:Ac reduced the moth catches. Therefore the optimum range of Z11-16:Ac seemed to be 15 to $150 \mu \mathrm{g} / \mathrm{septum}$ though there was no test below the $15 \mu \mathrm{g}$ level. 
Table 3. Catches of male black cutworm moths with glass capillary tubes $^{\mathrm{a}}$ dispensing $\mathrm{Z7-12:Ac}$ and $\mathrm{Z9-14:Ac}$ at various ratios

(Zentsuji, April 30-May 6, 1981)

\begin{tabular}{cccc}
\hline \multicolumn{3}{c}{ Ratio } & Total no. of males caught ${ }^{\mathrm{b}}$ \\
\cline { 2 - 3 } $\mathrm{Z} 7-12: \mathrm{Ac}$ & & $\mathrm{Z} 9-14: \mathrm{Ac}$ & $0 \mathrm{c}$ \\
\hline 0 & $:$ & 100 & $0 \mathrm{c}$ \\
10 & $:$ & 90 & $8 \mathrm{bc}$ \\
25 & $:$ & 75 & $21 \mathrm{~b}$ \\
50 & $:$ & 50 & $46 \mathrm{a}$ \\
75 & $:$ & 25 & $54 \mathrm{a}$ \\
90 & $:$ & 10 & $1 \mathrm{c}$ \\
100 & $:$ & 0 &
\end{tabular}

a ID: $0.266 \mathrm{~mm}$.

b The values indicated are the total for each 1 trap throughout the test period. The values followed by the same letter are not significantly different by Duncan's new multiple range test at $5 \%$ level. During the test period, 621 males obtained from the laboratory culture were released.

Table 4. Catches of male black cutworm moths with glass capillary tubes of various ID dispensing the 3:1 mixture of Z7-12:Ac and Z9-14:Ac

(Nonoichi, April 28-June 10, 1982)

\begin{tabular}{ccc}
\hline \multicolumn{2}{c}{ ID of glass tube $(\mathrm{mm})^{\mathrm{a}}$} & \multicolumn{1}{c}{ Total no. of males caught ${ }^{\mathrm{b}}$} \\
\hline 0.266 & $(0.056)$ & $56 \mathrm{ab}$ \\
0.376 & $(0.111)$ & $107 \mathrm{a}$ \\
0.532 & $(0.222)$ & $33 \mathrm{ab}$ \\
0.841 & $(0.556)$ & $7 \mathrm{~b}$ \\
\hline
\end{tabular}

a Values in the parentheses are the cross-sectional areas $\left(\mathrm{mm}^{2}\right)$ of the tubes.

b Values indicated are the total for 1 trap throughout the test period. Values followed by the same letter are not significantly different by DUNCAN's new multiple range test at $5 \%$ level.

A glass-capillary tube sealed at one end has been demonstrated to be a stable and long-lived dispenser of the synthetic sex pheromone for Agrotis segetum by Kozar and WAKAmura (1982). To evaluate the glass capillary formulation for A. ipsilon attractant, several tests were conducted. Firstly catches with mixtures of Z7-12: Ac and Z9-14:Ac at various ratios were investigated (Table 3 ). The maximum catch was obtained with the 90:10 (9:1) mixture but it was not significantly different from with 75:25 (3:1). Then the 3:1 mixture dispensed from glass tubes with various ID, or various crosssectional areas, were tested (Table 4 ). The greatest number of moths were attracted to the $0.376-\mathrm{mm}-\mathrm{Id}$ tube, but this catch was not significantly different from those to the 0.266- or 0.532-mm-ID tubes. This confirmed the result reported by HrLl et al. (1979).

The effect of the addition of Z11-16: Ac was evaluated in the case of the glasscapillary formulations. Z11-16:Ac was added to the 3:1 mixture of Z7-12:Ac and Z9-14:Ac and dispensed from glass tubes (Table 5). Z11-16:Ac and a 3:1 mixture of Z7-12:Ac and Z9-14:Ac were also dispensed from separate tubes that were held 
Table 5. Catches of male black cutworm moths with glass capillary tubes dispensing the mixture of Z7-12:Ac, Z9-14:Ac and Z11-16: Ac (Zentsuji, June 11-19, 1981)

\begin{tabular}{cccccc}
\hline \multicolumn{3}{c}{ Ratio } & \multicolumn{2}{c}{ ID of glass tube $(\mathrm{mm})^{\mathrm{a}}$} & Total no. of males caught $^{\mathrm{b}}$ \\
\cline { 1 - 3 } Z7-12:Ac & Z9-14:Ac & Z11-16:Ac & & \\
\hline 3 & 1 & 0 & 0.266 & $(0.056)$ & $22 \mathrm{a}$ \\
3 & 1 & 4 & 0.266 & $(0.056)$ & $20 \mathrm{a}$ \\
3 & 1 & 12 & 0.266 & $(0.056)$ & $11 \mathrm{ab}$ \\
3 & 1 & 36 & 0.266 & $(0.056)$ & $2 \mathrm{~b}$ \\
3 & 1 & 4 & 0.376 & $(0.111)$ & $11 \mathrm{ab}$ \\
3 & 1 & 12 & 0.532 & $(0.222)$ & $5 \mathrm{~b}$ \\
\hline
\end{tabular}

a Values in the parentheses are the cross sectional areas $\left(\mathrm{mm}^{2}\right)$ of the tubes.

b Values indicated are the total for 1 trap throughout the test period. Values followed by the same letter are not significantly different by Duncan's new multiple range test at $5 \%$ level.

Table 6. Catches of male black cutworm moths with separate glass capillary tubes held side-by-side dispensing a 3:1 mixture of Z7-12:Ac and Z9-14:Ac, and Z11-16:Ac

(Nonoichi, June 12-September 20, 1982)

\begin{tabular}{ccc}
\hline ID of glass tube of Z11-16:Ac $(\mathrm{mm})^{\mathrm{a}}$ & Total no. of males caught ${ }^{\mathrm{b}}$ \\
\hline- & $(0.222)$ & $91 \mathrm{a}$ \\
0.532 & $(0.556)$ & $53 \mathrm{ab}$ \\
0.841 & $(1.11)$ & $12 \mathrm{~b}$ \\
$0.841 \times 2 \mathrm{c}$ & $1 \mathrm{~b}$ \\
\hline
\end{tabular}

a Basic formulation: a 0.266-mm-ID glass tube dispensing the $3: 1$ mixture of $\mathrm{Z7}-12$ :Ac and Z9-14:Ac. Values in the parentheses are cross-sectional area $\left(\mathrm{mm}^{2}\right)$ of tubes of $\mathrm{Z11-16:Ac}$.

b Values indicated are total for 1 traps throughout the test period. Values followed by the same letter are not significantly different by Duncan's new multiple range test at $5 \%$ level.

c Two tubes of $0.841-\mathrm{mm}$-ID were held side-by-side.

Table 7. Effective duration of rubber-septum formulation of sex attractant for male black cutworm (Fujishima, 1984)

\begin{tabular}{|c|c|c|c|c|c|c|}
\hline \multirow{2}{*}{ Lure } & \multicolumn{6}{|c|}{ Total no. of males caught ${ }^{\mathrm{a}}$} \\
\hline & $\begin{array}{l}\text { April } 23- \\
\text { May } 9\end{array}$ & $\underset{29}{\text { May } 10-}$ & $\begin{array}{l}\text { May } 30- \\
\text { June } 19\end{array}$ & $\begin{array}{l}\text { June } 20- \\
\text { July } 10\end{array}$ & $\begin{array}{l}\text { July } 11- \\
\text { Aug. } 16\end{array}$ & $\begin{array}{l}\text { Aug. } 17- \\
\text { Sept. } 16\end{array}$ \\
\hline $\begin{array}{l}\text { Rubber without } \\
\text { replacementb }\end{array}$ & 16 & 15 & 7 & 7 & 3 & 0 \\
\hline $\begin{array}{l}\text { Rubber with } \\
\text { replacement } t^{b, c}\end{array}$ & 16 & 7 & 0 & 25 & 6 & 17 \\
\hline Glass capillary tube & 1 & 0 & 0 & 1 & 10 & 3 \\
\hline Black light & 0 & 0 & 0 & 8 & 17 & 0 \\
\hline
\end{tabular}

a Values indicated are the totals for 1 trap throughout the presented periods.

b A rubber septum was impregnated with $30 \mu \mathrm{g}$ of Z7-12:Ac, $10 \mu \mathrm{g}$ of Z9-14:Ac and $150 \mu \mathrm{g}$ of Z11-16:Ac.

c Rubber septa were replaced at the beginning of each indicated period.

d A 0.266-mm-ID glass capillary tube dispensing a 3:1 mixture of Z7-12:Ac and Z9-14:Ac. 
side-by-side (Table 6). In both cases, catches of males were reduced as increasing amounts of Z11-16: Ac were dispensed. The larger concentrations of Z11-16: Ac seemed to have an inhibitory effect on the attraction of males when dispensed from glass-capillary tubes. It is not known why the synergistic effect of Z11-16: Ac observed on the rubberseptum formulations becomes an inhibitory effect in the glass-capillary formulations.

The effective duration of the dispensers was determined with a rubber septum impregnated with $30 \mu \mathrm{g}$ of Z7-12:Ac, $10 \mu \mathrm{g}$ of Z9-14:Ac, and $150 \mu \mathrm{g}$ of Z11-16:Ac and a 0.226-mm-ID glass tube dispensing 3:1 mixture of Z7-12:Ac and Z9-14:Ac (Table 7). Two traps were used for the rubber-septum formulation; one was maintained without replacement of the lure throughout the test period, and the other was with replacement of the lure at 20- to 40-day intervals. For the comparison, a black light trap was also used. The catches with rubber septum remained high without replacement from April 23 to June 19 and then decreased. Part of the decrease may be due to the loss of attractant and/or the shift of the ratio of its components. The effective duration seemed to be about 2 months. However, daily trap counts showed that, compared with the original septum, fewer moths were attracted to the replaced septum during the first 10 days of exposure in the trap. Practically, this formulation should have a 10-day pre-exposure before setting in a trap, and then it will be effective as a lure for at least 1 month. Although the effective duration of the glass tube seemed more than 5 months, catches were too low to obtain a seasonal prevalences of the black cutworm moth expecially during the first flight.

The relative differences in the catches with the attractant and black light traps may have been dependent upon the relative locations of the two types of traps and this will be the subject of another study. Nevertheless, these results indicate that the attractant-baited traps are generally more efficient than light traps, and will be useful for population monitoring for early warning of sudden outbreaks.

It has not been confirmed whether Z11-16:Ac is the actual pheromone component produced by female black cutworm moth.

\section{ACKNOWLEDGEMENTS}

We are grateful to G. L. Ayre, Research Station, Agriculture Canada, Winnipeg, Manitoba, Dr. E. STÄDler, Swiss Federal Research Station, CH-8820 Wädenswil, Switzerland, and Dr. R. A. Galbreath, Entomology Division, D.S.I.R., Auckland, New Zealand, for the allowance to cite their unpublished data, and Dr. S. TAKahashi, Pesticide Research Institute, Kyoto University, for kindly supplying the synthetic compound. We also wish to thank Dr. Y. TAmaki of the National Institute of Agro-Environmental Sciences, for critically reading the manuscript.

\section{REFERENCES}

Duncan, D. B. (1955) Multiple range and multiple $F$ test. Biometrics 11: 1-42.

Hill, A. S., R. W. Rings, S. R. Swier and W. L. Roelofs (1979) Sex phermone of the black cutworm moth, Aqrotis ipsilon. J. Chem. Ecol. 5: 439-457.

Kozai, S. and S. Wakamura (1982) Sex pheromone of the turnip moth, Aqrotis segetum Denis et SaHiffermüller (Lepidoptera: Noctuidae): Glass capillary formulation of synthetic sex pheromone. Jap. J. appl. Ent. Zool. 26: 262-265 (in Japanese with an English summary).

Ohmori, H. and T. HASEgawa (1968) Unusual outbreaks of the black cutworm on the tame pasture in Tohoku district in 1967. Shokubutsu-Boeki 22: 162-164 (in Japanese). 